\title{
Comparison of Linear and Non-linear 2D+T Registration Methods for DE-MRI Cardiac Perfusion Studies
}

\author{
Gert Wollny and María-Jesus Ledesma-Carbayo
}

\begin{abstract}
A series of motion compensation algorithms is run on the challenge data including methods that optimize only a linear transformation, or a non-linear transformation, or both - first a linear and then a non-linear transformation. Methods that optimize a linear transformation run an initial segmentation of the area of interest around the left myocardium by means of an independent component analysis (ICA) $\left(I C A-^{*}\right)$. Methods that optimize non-linear transformations may run directly on the full images, or after linear registration. Non-linear motion compensation approaches applied include one method that only registers pairs of images in temporal succession $(S E R I A L)$, one method that registers all image to one common reference (AllToOne), one method that was designed to exploit quasi-periodicity in free breathing acquired image data and was adapted to also be usable to image data acquired with initial breath-hold ( $Q U A S I-P$ ), a method that uses ICA to identify the motion and eliminate it $(I C A-S P)$, and a method that relies on the estimation of a pseudo ground truth $(P G)$ to guide the motion compensation.
\end{abstract}

\section{Introduction}

Various motion compensation methods are presented that are applied to the first-pass gadolinium-enhanced myocardial perfusion data provided in the motion compensation challenge. All these methods were implemented by using a freely available toolkit for gray scale image processing MIA [1].

The motion compensation schemes include methods that (1) use an independent component analysis (ICA) to segment the area of interest around the left myocardium, and to identify motion and eliminate it [2], (2) a method, QUASI-P, initially designed for the application to free breathing data [3], but here adapted to be applicable to data acquired with initial breath hold, (3) two generic methods, SERIAL and AllToOne, that only require that the data to comprise intra-subject tracking of movement, and (4) a method that uses pseudo ground truth $(P G)$ to guide the motion compensation [4].

The ICA-based methods can be run with both - linear and non-linear registration, all other methods rely on non-linear registration to achieve motion 
compensation. In the following the image registration back-end and methods are described in more detail.

\section{Methods}

\section{$2.1 \quad$ Image Registration}

Given a $d$-dimensional domain $\Omega \subset \mathbb{R}^{d}$ and a space of images $\mathbb{I}=\{I \mid I: \Omega \rightarrow \mathbb{R}\}$, and given a study image $S \in \mathbb{I}$ and a reference image $R \in \mathbb{I}$, registration aims at transforming the study image $S$ with respect to the reference image $R$, so that structures at the same coordinates in both images represent the same object. In practice, given a space of allowed transformation $\mathbb{T}$ this is achieved by finding a transformation $T_{\text {reg }} \in \mathbb{T}$ that minimizes a given cost function $F_{\text {cost }}: \mathbb{I} \times \mathbb{I} \rightarrow$ $\mathbb{R}$, while constraining the transformation through the joint minimization of an energy term $E: \mathbb{T} \rightarrow \mathbb{R}:$

$$
T_{\text {reg }}:=\arg \min _{T \in \mathbb{T}}\left(F_{\text {cost }}\left(S_{T}, R\right)+\kappa E(T)\right)
$$

The cost function $F_{\text {cost }}$ accounts for the mapping of similar structures. $E(T)$ ensures topology preservation, which is necessary to maintain structural integrity in the study image, and it thus introduces a smoothness constraint on the transformation $T$. The parameter $\kappa$ is a weighting factor that balances registration accuracy and transformation smoothness.

Based on the allowed transformation space $\mathbb{T}$ one can distinguish between linear and non-linear registration. With linear registration, $\mathbb{T}$ the topology is always preserved, hence the additional energy term $E(T)$ is not required; consequently $\kappa$ is set to zero in this case. In non-linear registration, this preservation of topology is not guaranteed and it is advisable to add an energy term $E(T)$ with a positive weight $\kappa$.

Cost Functions. In our application, the cost function $F$ is derived from a so-called voxel-similarity measure that takes into account the intensities of the whole image domain. As a consequence, the driving force of the registration is calculated directly from the given image data.

Specifically, we employ three image similarity measures:

1. The sum of squared differences (SSD)

$$
F_{\mathrm{SSD}}(S, R):=\frac{1}{2} \int_{\Omega}(S(\mathbf{x})-R(\mathbf{x}))^{2} d \mathbf{x}
$$

2. normalized gradient fields (NGF) as given in [3]; i.e. with the image noise level $\eta$ and $\epsilon$ a measure for boundary "jumps" (locations with a high gradient) that is defined as

$$
\epsilon:=\eta \frac{\int_{\Omega}|\nabla I(\mathbf{x})| d \mathbf{x}}{\int_{\Omega} d \mathbf{x}}
$$


and the normalized gradient

$$
\mathbf{n}_{\epsilon}(I, \mathbf{x}):=\frac{\nabla I(\mathbf{x})}{\sqrt{\sum_{i=1}^{d}(\nabla I(\mathbf{x}))_{i}^{2}+\epsilon^{2}}},
$$

the NGF cost function for images of dimension $d$ is defined by

$$
F_{\mathrm{NGF}}(S, R):=\frac{1}{2} \int_{\Omega}\left(\left\|\mathbf{n}_{\epsilon}(R, x)\right\|^{2}-\frac{\left\langle\mathbf{n}_{\epsilon}(R, x), \mathbf{n}_{\epsilon}(S, x)\right\rangle^{2}}{\left\|\mathbf{n}_{\epsilon}(R, x)\right\|\left\|\mathbf{n}_{\epsilon}(S, x)\right\|}\right)^{2} d \mathbf{x},
$$

3. localized normalized cross correlation (LNCC)

$$
F_{\mathrm{NGF}}(S, R, W):=\frac{1}{\# \Omega} \sum_{\mathbf{x} \in \Omega} n c c(S, R, \mathbf{x}, W)
$$

with $k$ defining the neighborhood window $W:=\{-k, k\}^{2}$ on which the cross correlation is evaluated according to

$$
n c c(S, R, \mathbf{x}, W):=\frac{\left(\sum_{\mathbf{d} \in W} R(\mathbf{x}+\mathbf{d}) S(\mathbf{x}+\mathbf{d})\right)^{2}}{\sum_{\mathbf{d} \in W} R^{2}(\mathbf{x}+\mathbf{d}) \sum_{\mathbf{d} \in W} S^{2}(\mathbf{x}+\mathbf{d})}
$$

SSD can be used when study and reference image exhibit similar intensity distributions - e.g. when synthetic references are used for registration, and NGF and LNCC are used otherwise. Here, NGF and LNCC have an advantage over statistical measures like mutual information, since they are truly local similarity measure that can accommodate the local intensity change induced by the contrast agent passing through the heart ventricles and the myocardium. In addition, NGF has the advantage that it is fairly easy to implement and as a low computational cost.

Transformation Spaces. For linear registration we restrict the transformation space to either translations only, or to rigid transformations (i.e. translations and rotations), or affine transformations.

For non-linear registration methods the transformation space $\mathbb{T}$ is restricted to transformations that can be described by a B-splines based model [5], and the regularization $E$ is based on the separate norms of the second derivative of each of the deformation components [6]. The balance between smoothness of the resulting transformation and the amount of non-rigidity that allows for the registration of smaller features can be fine tuned by setting the B-spline coefficient rate $c_{\text {rate }}$ and the weighting factor $\kappa$ accordingly. For both parameters, higher values result in smoother transformations that preserve the per-voxel volume better but come at the cost of a reduced ability to register small features.

\subsection{Motion Compensation Schemes}

Independent Component Analysis Based Schemes. Various of the applied motion compensation schemes make use of independent component analysis to 
create synthetic, motion-free references to guide the registration. Here a multipass approach is taken: In the first step, an ICA is run. Then the resulting components are classified based on a wavelet analysis of the mixing matrix, and the component representing motion is discarded. In addition, in the first pass the left and right ventricle cavity is identified and segmented, and the region of interest containing the left ventricle myocardium is extracted to restrict the following registration to this area. Synthetic reference images are then created by mixing the motion-free components, and the original images are registered to these references by optimizing SSD. Since initially the synthetic references are rather blurry, the method is run various times to achieve full motion compensation. The details of this method are described in [2], and a minor adjustment to identify a failed segmentation based on the size of the heart was added [7].

In its original implementation all images would be registered to a mean of the breathing motion that does normally not correspond to a specific image of the original series. Therefore, in order to accommodate the requirement of the challenge that all images need to be aligned to a predefined reference image, the algorithm was changed so that in each pass the registration of the reference image was inverted, i.e. the synthetic reference was registered to its corresponding original image, and the resulting transformation is applied to all other registered images.

This method was applied correcting for translations only (ICA-T), thereby mimicking [8], correcting a rigid transformation $(I C A-R)$, an affine transformation $(I C A-A)$, and a B-Spline based transformation $(I C A-S P)$. In addition, we run combinations of these ICA-based linear and non-linear motion compensation schemes, i.e. $I C A-T+S P$ and $I C A-A+S P$.

Note, that this method was originally designed to be applied to data acquired free breathing, but synthetic experiments indicated that the method can also be applied to data that was acquired with initial breath hold [2].

$Q U \boldsymbol{A} S \boldsymbol{I}-\boldsymbol{P}$. The algorithm implements the method proposed in [3] that was initially designed to be run on free breathing aquired data. Here, the quasiperiodicity of free breathing was used to identify key frames that are already closely aligned, and registered using NGF to an automatically chosen reference frame. Then, by linearly combining these pre-aligned images synthetic references are created and the remaining images are registered to their synthetic counterparts by optimizing SSD.

Since five series the data provided for the challenge was acquired with initial breath hold, and the challenge rules require that all images are aligned to a predefined reference the algorithm was changed in two aspects: Firstly, instead of estimating the global reference image automatically, it was set, and secondly, when creating the initial subset it was made sure that the temporal distance between two consecutive images was not larger than seven acquisition frames. Without such a limitation it may happen that the algorithm does not add enough time points to the initial set, resulting in a failure of the second registration step that results from badly created synthetic references. 
Generalized Non-linear Motion Compensation Schemes. We also run registration schemes that make no assumptions about the data other than that it comprises an intra-subject tracking of movement. Specifically, two approaches were run:

SERIAL: Here, only images in temporal succession are registered and then the transformations are applied accumulated. As registration criterion we used a weighted combination of NGF and SSD as proposed in [9].

AllToOne: With this method we register all images to one global reference by using $\mathrm{LNCC}$ as registration criterion.

In both cases, a B-spline transformation was optimized.

Pseudo Ground Truth. This method considers the estimation of a pseudo ground truth $(P G)[4]$ in order to create synthetic reference images that are then used to compensation for the motion. This algorithm requires the images to be already linearly registered, otherwise the estimation of the pseudo ground truth will fail [2]. Hence we first run an ICA-based linear motion compensation scheme then followed by the $P G$. The implementation of the estimation of $P G$ we used differs somewhat from the algorithm described in [4]. Specifically, instead of using a demons based registration scheme we use the same B-Spline based method for image registration as given above, and instead of using Gaussian elimination to solve the PG estimation problem, we used the L-BFGS algorithm ([10]). Like the ICA-based methods, this algorithm is also run in a multi-pass scheme.

In summary, the combined motion compensation algorithm we run here are $I C A-T+P G$ and $I C A-A+P G$.

\section{Experiments}

Above registration schemes were applied to the provided challenge data. For the various methods the registration parameters were set as follows:

For optimization of linear transformations we used the simplex algorithm [11] (breaking condition for the simplex algorithm was set to 0.01 , and its start step size to 0.001). For non-linear registrations run with the ICA-based schemes and PG we used rank-1 method of the shifted limited-memory variable metric algorithm (VAR1) [12] as optimizer. In all other cases a gradient descent optimizer was applied. For non-linear registrations with multi-pass schemes we used an initial B-spline knot spacing of 16 and a regularization weight $\kappa$ of 10 . With each new pass, these quantities were divided by 2 . For all multi-pass schemes at most three registration passes were run. With AllToOne, QUASI-P, and SERIAL, we set the B-spline knot spacing to 5 and the regularization weight $\kappa$ to 0.1 .

For best reproducibility the experiments were obtained by running the motion compensation within a virtualized Ubuntu Linux 14.04 (i386) environment that can be downloaded as virtual hard disk [13], but with MIA [1] updated to version 2.2.2. The scripts used to run the experiments are made available at https://sourceforge.net/projects/mia/files/Scripts/ 


\section{Results}

Visual inspection of the results show a mixed picture. No method provides a visually pleasing motion compensation for all cases.

Specifically, in many cases QUASI-P fails to provide motion compensation at the beginning of the series and even deforms the image area containing the LV myocardium beyond recognition (Fig. 1 (a)), a problem with QUASI-P that that can be attributed to the generation of synthetic references by using linear interpolation between time steps [3]. Similar registration errors can be observed to a lesser extend by using the AllToOne approach (Fig. 1 (b)).

The identification of the LV and RV cavities, and consequently, the segmentation of a ROI around the LV myocardium failed for the data sets 1,3,5, and 9 aquired under stress, as well as for the un-gated data. Here, linear registration

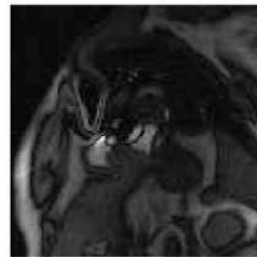

(a) QUASI-P 8/16 Rest

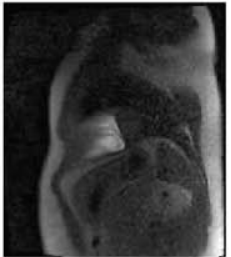

(b) AllToOne $2 / 7 \mathrm{Str}$.

Fig. 1. QUASI-P sometimes not only fails to achieve motion compensation, but also results in a deformation of key images of the series (a). Likewise, AllToOne also may result in such deformations when registering images at the beginning of the series (b), but the frequency of such occurrences is lower than with QUASI-P.

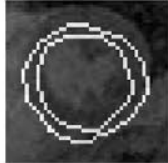

(a) original

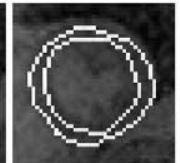

(b) AllToOne

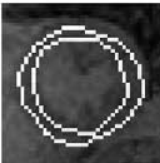

(c) QUASI-P

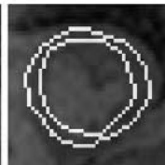

(d) SERIAL

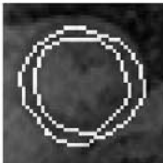

(e) ICA-T+SP

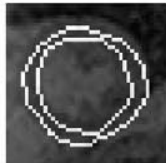

(f) ICA-R+SP

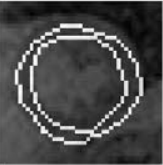

(g) ICA-A+SP

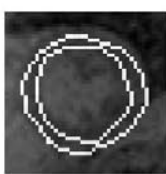

(h) ICA-SP

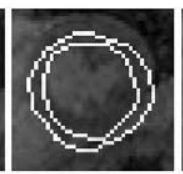

(i) $\mathrm{ICA}-\mathrm{T}+\mathrm{PG}$

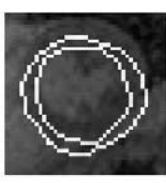

(j) $\mathrm{ICA}-\mathrm{R}+\mathrm{PG}$

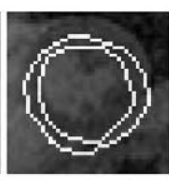

(k) ICA-A+PG

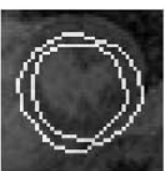

(1) ICA-T

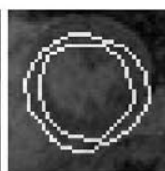

(m) ICA-R

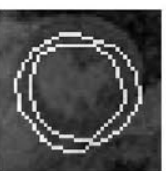

(n) ICA-A

Fig. 2. Data set 5 (stress): Results for slice 50 (cropped) and all applied methods. The linear registration based methods (l-m) because the ROI around the myocardium could not be segmented for this data set. Running PG as additional non-linear registration (i-k) after a failed linear registration does also not result in an alignment as good as with all the other methods. 


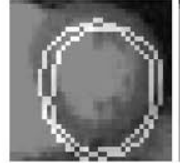

(a) original

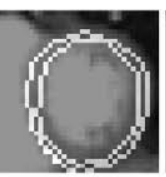

(h) ICA-SP

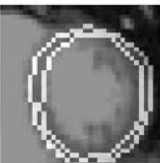

(b) AllToOne

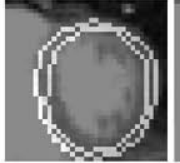

(c) QUASI-P

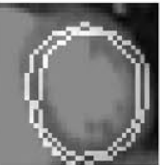

(d) SERIAL

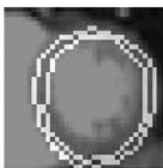

(e) ICA-T+SP

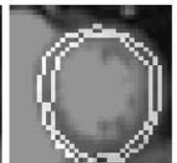

(f) $\mathrm{ICA}-\mathrm{R}+\mathrm{SP}$

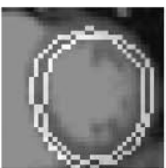

(g) ICA-A+SP

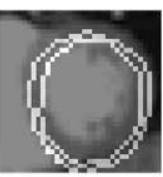

(i) $\mathrm{ICA}-\mathrm{T}+\mathrm{PG}$

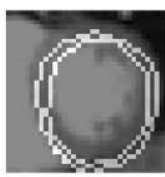

(j) ICA-R+PG

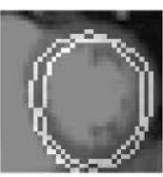

(k) ICA-A+PG

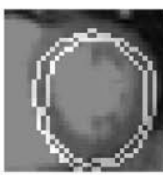

(1) ICA-T

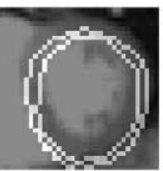

(m) ICA-R

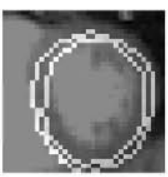

(n) ICA-A

Fig. 3. Data set 8 (stress): Results for slice 45 (cropped) and all applied methods. Here, AllToOne (b) and QUASI-P (c) and all ICA*SP methods (e-h) show a good alignment for this slice, whereas the other methods that include non-linear registration (d, i-k) this alignment is not as good, but still better than without correction (a). Applying only linear registration (l-n) shows a still notable but small improvement over the uncorrected data (a) for this slice.

methods ICA-T, ICA-R, and ICA-A could not provide any notable motion compensation which is illustrated in the example images in Fig. $2(\mathrm{l}-\mathrm{m})$. Since the ICA-*-PG methods require initial linear registration, the also failed to provide notable motion compensation for these data sets.

As another example we present slice 45 from data set 8, stress (Fig. 3). Here the picture is a bit different: AllToOne, QUASI-P and all the combinations utilizing ICA-SP (either solely, or with initial linear registration) provide visually good results, While the other methods provide still provide a better alignment than without motion compensation, judging only from visual inspection the improvement achieved by only running linear registration is quite small.

The obtained avarage MBF values corrected for the AIF are reported below, in Table 1 for the rest studies, and in Table 2 for the stress studies. The values were obtained by running the according Matlab scripts provided by the challenge organizers and evaluating the average of the per section MBF values.

We also applied all methods to the ungated data. The segmentation of the LV region with the ICA based methods failed, and consequently no motion compensation was achieved by applying linear registration methods only. ICA-SP provided visually pleasing results, AllToOne, QUASI-P, and SERIAL provide a mixed picture over the time series, introducing rather erratic deformations in the images in parts of the series. Running GT (after the failed linear registration) also provided motion compensation. 
Table 1. Average MBF values for the rest studies, and the ungated study

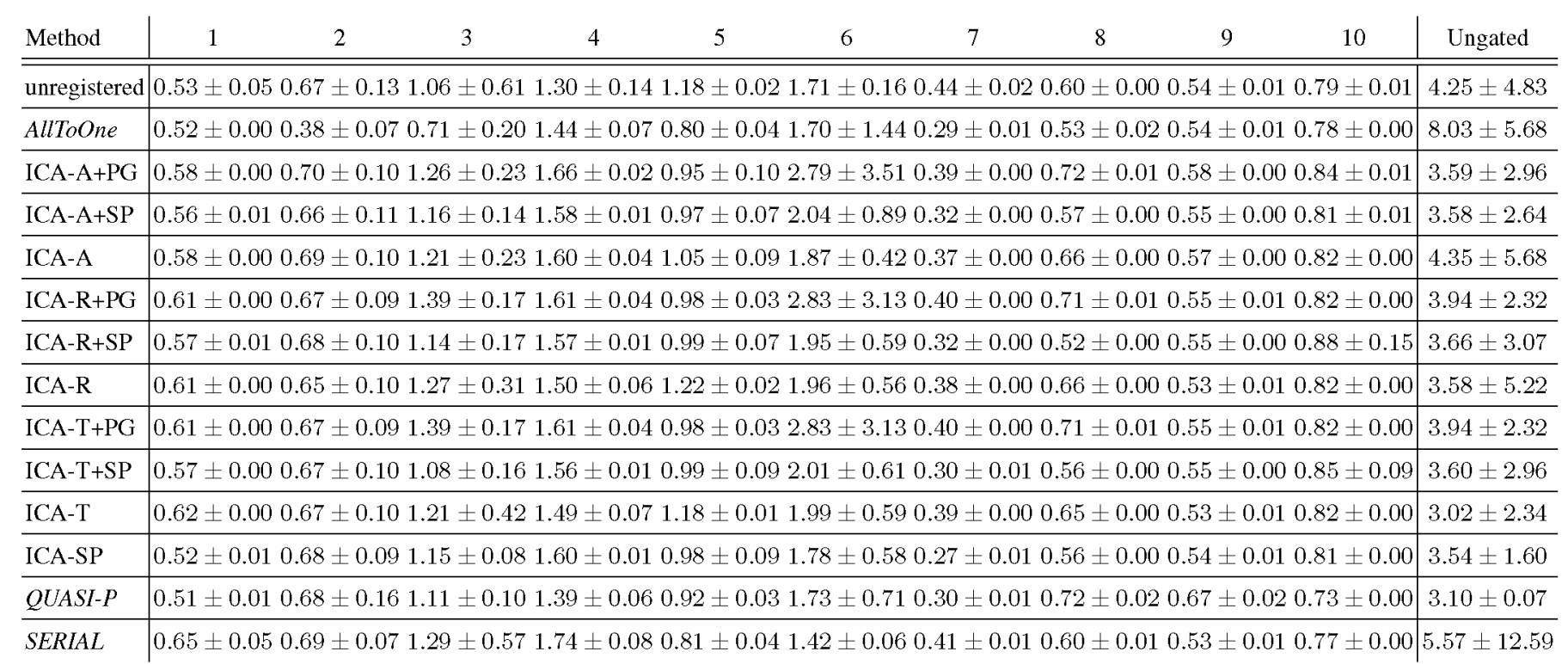


Table 2. Average MBF values for the stress studies

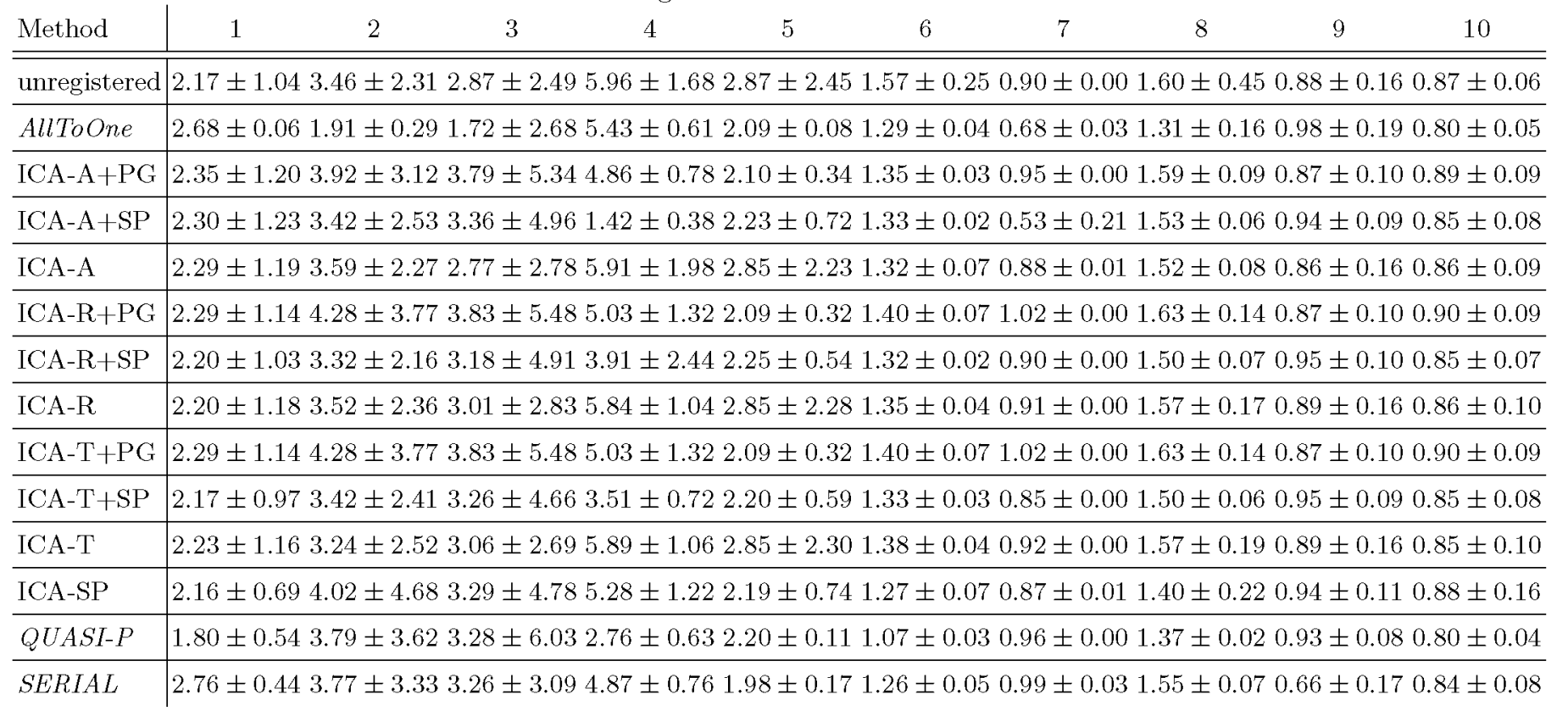




\section{Conclusion}

We applied a series of 13 motion compensation methods based on image registration to the challenge data, out of these two (AllToOne and SERIAL) a generalized registration methods, and QUASI-P and all ICA based methods were specifically designed for free breathing data. The ICA based methods provide a method to identify and segment the heart ventricular cavities, and therefore they provide the possibility to restrict the registration to this area making the application of linear registration methods possible. If this segmentation failed, however, linear registration could no provide motion compensation, since the non-moving body parts in the image dominate. The linear methods were also combined with a ICA based and a PG based non-linear registration method. Both provided additional motion compensation but in the cases when linear registration failed the ICA based method usually provided visually better motion compensation than the PG method.

The main advantage of non-linear registration is that registrations is independent of an initial segmentation of the ROI. Also with ungated data, the additional deformation of the beating heart can only be captured by non-linear registration. Linear registration, on the other hand, is faster and is not not prone to registration errors like can be seen in fig. 1.

\section{References}

1. Wollny, G., Hublin, J., Ledesma-Carbayo, M.J., Skinner, M., Kellman, P., Hierl, T.: MIA - A Free and Open Source Software for Gray Scale Medical Image Analysis. Source Code for Biology and Medicine 8:20 (2013)

2. Wollny, G., Kellman, P., Santos, A., Ledesma-Carbayo, M.J.: Automatic Motion Compensation of Free Breathing acquired Myocardial Perfusion Data by Using Independent Component Analysis. Medical Image Analysis 16(5), 1015-1028 (2012)

3. Wollny, G., Ledesma-Carbayo, M.J., Kellman, P., Santos, A.: Exploiting Quasiperiodicity in Motion Correction of Free-Breathing Myocardial Perfusion MRI. IEEE Trans. Med. Imag. 29(8), 1516-1527 (2010)

4. Li, C., Sun, Y.: Nonrigid registration of myocardial perfusion MRI using pseudo ground truth. In: Yang, G.-Z., Hawkes, D., Rueckert, D., Noble, A., Taylor, C. (eds.) MICCAI 2009, Part I. LNCS, vol. 5761, pp. 165-172. Springer, Heidelberg (2009)

5. Kybic, J., Unser, M.: Fast Parametric Elastic Image Registration. IEEE Trans. Image Process. 12(11), 1427-1442 (2003)

6. Rohlfing, T., Maurer Jr., C.R., Bluemke, D.A., Jacobs, M.A.: Volume-Preserving Nonrigid Registration of MR Breast Images Using Free-form Deformation with an Incompressibility Constraint. IEEE Trans. Med. Imag. 22, 730-741 (2003)

7. Wollny, G., Kellman, P.: Free breathing myocardial perfusion data sets for performance analysis of motion compensation algorithms. GigaScience 3:23 (2014)

8. Gupta, V., Hendriks, E., Milles, J., Van Der Geest, R., Jerosch-Herold, M., Reiber, J., Lelieveldt, B.: Fully Automatic Registration and Segmentation of Firstpass Myocardial Perfusion MR Image Sequences. Academic Radiology 17(11), $1375-1385(2010)$ 
9. Wollny, G., Ledesma-Carbayo, M.J., Kellman, P., Santos, A.: A new similarity measure for non-rigid breathing motion compensation of myocardial perfusion MRI. In: Proc. of the 30th Int. Conf. of the IEEE-EMBS, Vancouver, BC, Canada, pp. 3389-3392 (2008)

10. Fletcher, R.: Practical Methods of Optimization, 2nd edn. Wiley (2000)

11. Nelder, J., Mead, R.: A Simplex Method for Function Minimization. Computer Journal 7, 308-313 (1965)

12. Vlcek, J., Luksan, L.: Shifted limited-memory variable metric methods for largescale unconstrained minimization. J. Computational Appl. Math. 186, 365-390 (2006)

13. Wollny, G., Kellman, P.: Supporting material for: Free breathing myocardial perfusion data sets for performance analysis of motion compensation algorithms. GigaScience, Database (2014). http://dx.doi.org/10.5524/100106 